\title{
Making ambiguous displays unambiguous: The influence of real colors and colored aftereffects on perceptual alternation
}

\author{
JACK BROERSE \\ Brisbane College of Advanced Education, Mount Gravatt, Queensland, Australia \\ and \\ BORIS CRASSINI \\ University of Queensland, St. Lucia, Queensland, Australia
}

\begin{abstract}
The relationship between orientation-contingent colored aftereffects (CAEs) and perceptual alternation of ambiguous displays was investigated in three experiments. In all experiments, the ambiguous test display consisted of vertical and horizontal contours that either could be perceived as separate surfaces (the diamond organization) or could be combined to form upright and inverted Us (the rectangles organization). In Experiments 1 and 2, observers inspected the test display when it was achromatic, when it was colored in a manner consistent with the appearance of CAEs, and when it was colored in a non-CAE manner. In Experiment 3, the test display was inspected monocularly before and after monocular color-orientation adaptation. The achromatic viewing conditions of Experiments 1 and 2, both monocular preadaptation achromatic viewing conditions of Experiment 3, and the postadaptation achromatic viewing condition involving the nonadaptation eye in Experiment 3 produced essentially the same results: reports of approximately equal duration for the diamond and rectangles organizations and steady rates of perceptual alternation. The non-CAE color conditions of Experiment 2 produced a similar pattern of results. However, the color condition of Experiment 1 (i.e., simulated CAEs), and the postadaptation condition involving the adaptation eye in Experiment 3 produced a decrement in the perceptual alternation rate, with the diamond organization being reported almost exclusively. These results indicate that ambiguous displays can be made unambiguous by altering the appearance of the displays either through the addition of appropriate colors or by exposing observers to appropriate adaptation conditions. These results are consistent with stimulus-bound explanations of both CAEs and perceptual alternation.
\end{abstract}

The two displays in Figure 1 are perceptually ambiguous. That is, although the physical properties of the displays (e.g., the orientation, contrast, and number of contours within the displays) do not change during a period of observation, perception of the displays does change. On some occasions, perception of Figure la (the Schroeder staircase) is such that the point farthest from the observer appears to be the upper right-hand corner. On other occasions, the perceived staircase is such that this same point appears to be the nearest point relative to the observer. Similarly, Figure 1b may be seen as consisting of a centrally located, diamond-shaped figure made up of horizontal bars superimposed on a background of

A preliminary presentation of the results reported here was made at the Tenth Experimental Psychology Conference held in May 1983 at the University of Tasmania, Australia. We gratefully acknowledge the expert technical assistance of Jack Pierce in setting up the apparatus. Requests for reprints should be sent to either Jack Broerse, Department of Psychology and Special Education, Brisbane College of Advanced Education, P.O. Box 82, Mount Gravatt, Queensland 4122, Australia, or Boris Crassini, Department of Psychology, University of Queensland, St. Lucia, Queensland 4067, Australia. vertical bars; an alternative organization involves both the horizontal and vertical bars forming upright and inverted concentric $U$ shapes. The first perceptual organization of Figure $1 \mathrm{~b}$ is termed the diamond organization, and the second is termed the rectangles organization in the remainder of this report.

Ambiguous displays such as Figures $1 \mathrm{a}$ and $1 \mathrm{~b}$ are intrinsically interesting phenomena, and perceptual ambiguity has been incorporated into the works of a number of artists (see Escher, 1961; Gregory \& Gombrich, 1973). However, theoretical importance has also been attributed to ambiguous displays, in particular with respect to what such phenomena indicate about perception in general. For example, the fluctuations in perceptual organization that occur during inspection of displays such as Figures $1 \mathrm{a}$ and $\mathrm{lb}$ have been taken as supporting evidence for indirect theories of perception (see Fodor \& Pylyshyn, 1981; Gregory, 1974; Oatley, 1978). Proponents of indirect theories argue that the sensory data forming the basis of perception are fundamentally ambiguous and require interpretation by some central cognitive process before meaningful perception can occur. In these 


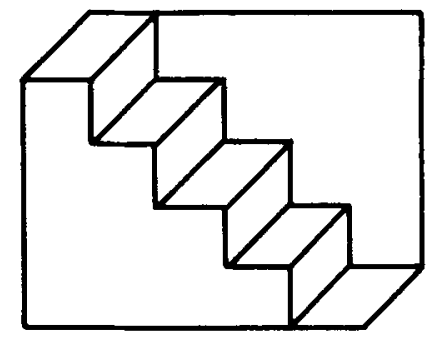

(a)

Figure 1. Examples of ambiguous displays. The Schroeder staircase (a) and the diamond/rectangles figure used as a test stimulus in CAE studies (b).

terms, perception may be stimulus driven, but in its essence it is not stimulus bound. Instead, perception is the outcome of the imposition of order (meaning) on sensory input by a cognitive process that is independent of the particular stimulus input (see Uttal, 1981, for a discussion of what he terms neorationalist theories of perception). Ambiguous displays are clear examples of this indirect or mediated view of perception in that, by definition, just such a process of imposition of meaning occurs during observation of ambiguous displays.

A somewhat different approach is taken in stimulusbound explanations of perceptual alternation. Attneave (1976), for example, reviewed a number of classic demonstrations of perceptual alternation and concluded that the simplest explanation of perceptual alternation was based on the idea that changes from one organization to another occurred when populations of neural mechanisms simultaneously simulated by the various components of the ambiguous displays became "fatigued or satiated or adapted" (p. 275). Such an explanation is in some respects similar, first, to Hering's (1874/1964) account of binocular rivalry (alternations in perceptual dominance produced by simultaneous presentation of, e.g., vertical contours to the left eye and horizontal contours to the right eye), and second, to more recent neurally based explanations of monocular rivalry (changes in perceptual dominance produced with monocular or binocular viewing of orthogonal contours; Stromeyer, 1978; Wade, 1975, 1977). For example, monocular rivalry occurs when vertical contours are superimposed on and intersect with horizontal contours: Sometimes the horizontal contours appear more salient, sometimes the vertical contours appear more salient, and sometimes both horizontal and vertical contours appear equally salient (resulting in a composite pattern of horizontal and vertical contours).

Subjective fluctuations between these three states (i.e., horizontal, vertical, composite horizontal and vertical)

have been attributed to fluctuations in the activity of "separate and not significantly overlapping populations of neurones sensitive to bar-gratings of particular orientations"' (Campbell, Gilinski, Howell, Riggs, \& Atkinson, 1973, p. 125; see also Stromeyer, 1978; Wade, 1977). Within this context, Morrone, Burr, and Maffei (1982) have suggested that inhibitory interactions between populations of nonoverlapping orientation-selective neurones (Benevento, Creutzfeldt, \& Kuhnt, 1972; Blakemore \& Tobin, 1972; Burr, Morrone, \& Maffei, 1981) may account for fluctuations in the activity of these populations during monocular rivalry.

We have suggested previously (Broerse \& Crassini, 1981) that the rectangles organization of Figure $1 \mathrm{~b}$ may be related to the composite organization reported during monocular rivalry. When the horizontal contours of Figure $\mathrm{lb}$ are equally salient, a unified horizontal and vertical organization (rectangles) is seen. But when the orthogonal contours are not equally salient, Figure $1 \mathrm{~b}$ appears segregated into two surfaces, an organization consistent with the diamond configuration. ${ }^{1}$ Considered in these terms, perceptual alternations in ambiguous displays like Figure $1 \mathrm{~b}$ may involve the same sorts of inhibitory interactions between nonoverlapping populations of neurones as those assumed to be involved in monocular rivalry (see Broerse \& Crassini, 1981, for a review of such figures).

The explanations of perceptual alternation proposed above are examples of what Uttal (1981) terms neoempiricist approaches to perception. Unlike neorationalist approaches, which emphasize the way in which cognitive factors mediate the perception of external stimulation, neoempiricist approaches emphasize the directness of the relationship between perception and stimulation. Perhaps a better term for the sorts of neurally based approaches described above is neuroempiricism. In considering the role of neural mechanisms, neuroempiricism not only preserves the general neoempiricist emphasis on stimulus-bound aspects of perception, but also serves as a contrast to other neoempiricist approaches that are indifferent to the role of neural mechanisms in perception (e.g., Gibson's direct theory of perception; see Gibson, 1979).

Two aspects of neuroempiricist analyses of perceptual alternation warrant comment. The first aspect relates to the identification of the ambiguous nature of ambiguous displays with the components of the displays. It is this type of analysis that lies at the basis of Gibson's (1979) rejection of ambiguous figures as having any significance in understanding perception of the natural, threedimensional environment. For Gibson, phenomena of perceptual alternations "are problems in their own right, independent of the problems of direct visual perception" (1979, p. 291). He points out that ambiguous displays like the Schroeder staircase are two-dimensional arrays (i.e., pictures) composed of lines and textures intended to simulate the structural information available in natural, threedimensional visual environments. The use of lines and tex- 
tures to simulate such information is the technique used by artists and painters to create representations of the real world (i.e., "as record of what its creator has seen or imagined, made available for others to see or imagine" [Gibson, 1979, p. 291]). In these terms, an ambiguous display is a picture that "requires two kinds of apprehension, a direct perceiving of the picture surface along with an indirect awareness of what it depicts"' (Gibson, 1979, p. 291). This indirect awareness of what is depicted (i.e., interpretation) is ambiguous because the information simulated in the picture surface is ambiguous. It follows, therefore, that as the information simulated in the picture becomes more realistic, perceptual ambiguity should decrease. $^{2}$

There is empirical evidence for the proposition that the perceptual ambiguity of ambiguous displays is altered by the addition of appropriate information. Virsu (1975), for example, made the Schroeder staircase display unambiguous by presenting it to subjects dichoptically with appropriate binocular disparity allowing only one perceptual organization to occur. Harris (1979) added texture gradients to the Schroeder staircase to produce a similar effect with binocular viewing. Lawson, Packard, Lawrence, and Whitmore (1977) varied the disparity information available during dichoptic viewing of Figure $1 \mathrm{~b}$. They found that both the diamond and rectangles organizations were reported by subjects under conditions of zero disparity. However, when the display was viewed under optimal conditions of crossed disparity, Lawson et al. (1977) found significant increases in the mean cumulative durations of the diamond organization. It is clear, then, that the ambiguous nature of displays such as Figures $1 \mathrm{a}$ and $1 \mathrm{~b}$ can be modified by changes in the component properties of the displays.

The second aspect of neuroempiricist analyses of ambiguous displays that warrants comment is the attribution of perceptual alternation to changes in the functioning of visual processing mechanisms within the observer. As outlined above, the neural mechanisms responding to particular components within an ambiguous display (e.g., the vertical contours of a monocular-rivalry display or of Figure 1b) become "satiated or adapted" (Attneave, 1976) during a period of observation. In so doing, their inhibitory influence on other mechanisms (e.g., those processing the horizontal contours of a monocular-rivalry display or of Figure 1b) decreases. Once free of this inhibition, the activity of these latter mechanisms determines what is perceptually dominant (e.g., horizontal contours in the case of monocular rivalry, or a diamond-shaped grating of horizontal contours in the case of Figure 1b).

An extension of this argument is that perceptual alternation should be modified by similar changes in visual processing mechanisms produced by stimulus events occurring prior to observation of the ambiguous displays. For example, Virsu (1975) demonstrated that adaptation to unambiguous Schroeder staircases influenced the appearance of ambiguous Schroeder staircases in a postadaptation test. During adaptation, Virsu (1975) presented un- ambiguous Schroeder staircases as either stereograms or three-dimensional models. In each case, only one of the two perceptual organizations commonly reported with Figure 1a was perceived. When an ambiguous test figure was viewed following such adaptation, the perceived organization reported was always opposite to the one observed during adaptation. Similar data have been reported following adaptation to unambiguous Necker cubes (Emmerson, 1979).

The results of the studies showing that perceptual organization of ambiguous displays can be changed by particular alternations in the components of the displays or by adaptation of the observer to unambiguous versions of the displays are consistent with stimulus-bound explanations of perception. Further support for this interpretation of perceptual alternation phenomena is provided by the data of Uhlarik, Pringle, and Brigell (1977), which indicate that the nature of an ambiguous display is altered following adaptation to stimuli not directly related to the ambiguous display.

Uhlarik et al. (1977) used an adaptation procedure designed to generate orientation-contingent colored aftereffects (CAEs) of the type first reported by McCollough (1965). Following adaptation to red-vertical and greenhorizontal gratings, subjects inspected a test stimulus similar to Figure $1 \mathrm{~b}$ and reported both perceptual alternations and the color appearance of the test display. Subjects reported CAEs (i.e., the vertical contours of the test display appeared green, and the horizontal contours red). However, the CAEs were not visible during the entire test period. They were most often reported by subjects during dominance of the diamond organization. In their discussion of the study, Uhlarik et al. (1977) emphasized the postadaptation changes in the color appearance of the test display rather than the postadaptation changes in perceptual organization. The latter aspect of Uhlarik et al.'s (1977) data was pointed out by Broerse and Crassini (1981), who showed (by reanalyzing their data) that, following adaptation, subjects reported mainly the diamond organization. That is, color-orientation adaptation resulted in orientation-contingent CAEs and a change in perceptual alternation.

Uhlarik et al. (1977) interpreted their correlational data in the following causal terms: The central mechanism responsible for determination of the dominant perceptual organization at time $t$ also determined the color appearance of the ambiguous display (i.e., determined the presence or absence of CAEs). An alternative interpretation is based on the proposition that the addition of color to particular regions of Figure $1 \mathrm{~b}$ acts in the same manner as the addition of appropriate binocular-disparity and texture-gradient information to other ambiguous displays. That is, the changes in perceptual organization and/or color appearance reported by Uhlarik et al. (1977) can be understood as resulting from the reduction in perceptual ambiguity prompted by the addition of color.

This reinterpretation of Uhlarik et al.'s data is consistent with our earlier proposals that the perceptual fluctu- 
ations reported during inspection of Figure $1 \mathrm{~b}$ and during inspection of monocular-rivalry displays may be related. When, say, red horizontals and green verticals are used in a monocular-rivalry display (instead of achromatic contours), reports of the composite organization (i.e., when horizontals and verticals are equally salient) occur only infrequently (Rauschecker, Campbell, \& Atkinson, 1973). If this composite organization is considered to be related to the rectangles organization of Figure $1 \mathrm{~b}$, then the presence of complementary aftereffects (colors contingent upon the orthogonal contours of Figure lb) would be expected to result in comparable decreases in reports of the rectangles organization. Of course, this interpretation is predicated on the further assumption that the "addition of color" can occur as a consequence of appropriate color adaptation of the observer rather than through actual changes in the chromatic properties of the display.

A partial test of the proposals outlined above would be the measurement of perceptual alternations with Figure $1 \mathrm{~b}$ modified by the addition of real colors selected to make Figure $1 \mathrm{~b}$ appear as it would if CAEs occurred. Although Uhlarik et al. (1977) used such a colored "ambiguous" display, they asked their subjects to report only changes in the color appearance of the display. The equally important issue of whether the presence of real colors alters perceptual organization (and rate of perceptual alternation) was not addressed.

The question of whether real or aftereffect colors influence the perceived organization of ambiguous test figures has been overlooked also in a number of other investigations involving the induction of CAEs (e.g., Jenkins \& Ross, 1977; Meyer \& Phillips, 1980; Milewski, Iaccino, \& Smith, 1980; see Broerse \& Crassini, 1981, for a review of these studies). In the three experiments to be reported below, we sought to rectify this situation by investigating the influence of real colors on perceptual organization (Experiments 1 and 2) and by measuring concurrently the color appearance and perceptual organization of an ambiguous test display following color-orientation adaptation (Experiment 3 ).

\section{EXPERIMENT 1}

Reanalysis of Uhlarik et al.'s (1977) data by Broerse and Crassini (1981) showed that, following adaptation, subjects report orientation-contingent CAEs and a change in perceptual alternations when viewing Figure $1 \mathrm{~b}$. To clarify the possible causal relationship between these two outcomes of adaptation, observers in Experiment 1 were required to report continuously, during a test period, their perceived organization of a display like Figure $1 \mathrm{~b}$ altered by the addition of colors selected to appear like CAEs. It was expected that the addition of these colors to the ambiguous test display would result in a decrease in perceptual alternation rate and an increase in the cumulative duration of diamond organization reports relative to mea- sures taken during inspection of an achromatic version of Figure $1 \mathbf{b}$.

\section{Method}

Subjects. Nine undergraduate student volunteers with normal or corrected-to-normal visual acuity served as observers. All were naive as to the purpose of the experiment and were screened for color vision defects using the Ishihara test. ${ }^{3}$

Materials and Apparatus. A black-and-white photographic print of Figure $1 \mathrm{~b}$ with matte finish was used as the test stimulus and was displayed on a matte black screen, as illustrated in Figure 2. The test display subtended $1.3^{\circ}$ (wide) $\times 2.8^{\circ}$ (high), and its spatial frequency was 4.2 cycles $/ \mathrm{deg}$. Two Kodak Carousel projectors (P1 and P2 in Figure 2) were fitted with 150-W quartz iodide filaments; a variable-aperture tachistoscope shutter was used to control luminance. Coloration of the test figure was achieved by using a composite color slide consisting of a circular red patch (Wratten Filter No. 26) embedded centrally in a green rectangle (Wratten Filter No. 55). When projected from Pl in sharp focus onto the ambiguous display, the red patch fell inside the central diamond of the test display such that the "sides" of the diamond were tangential to the perimeter of the red patch. Similarly, the perimeter of the outer green rectangle coincided with the perimeter of the test display. Defocusing Pl caused the red-green border to merge and become fuzzy. Phenomenally, defocusing Pl destroyed the circular form of the central red color patch so that color appeared to fill completely the central diamond. Neutral-density filters placed in front of P1 and white light projected onto the test display from P2 were used to desaturate the projected colors to levels commonly experienced during CAEs. ${ }^{4}$ In conditions in which the ambiguous test display was achromatic, luminance and contrast levels were adjusted to match approximately those of the colored condition.

The observers sat, with their heads supported on a chinrest, and viewed the stimulus display through a rectangular aperture in a matte black screen which obscured the projectors and other extraneous details of the apparatus and its surroundings (see Figure 2). Two response buttons were mounted on a panel so that the observers could depress separate buttons when each of the figural organizations that could be experienced were dominant. The state (i.e.,
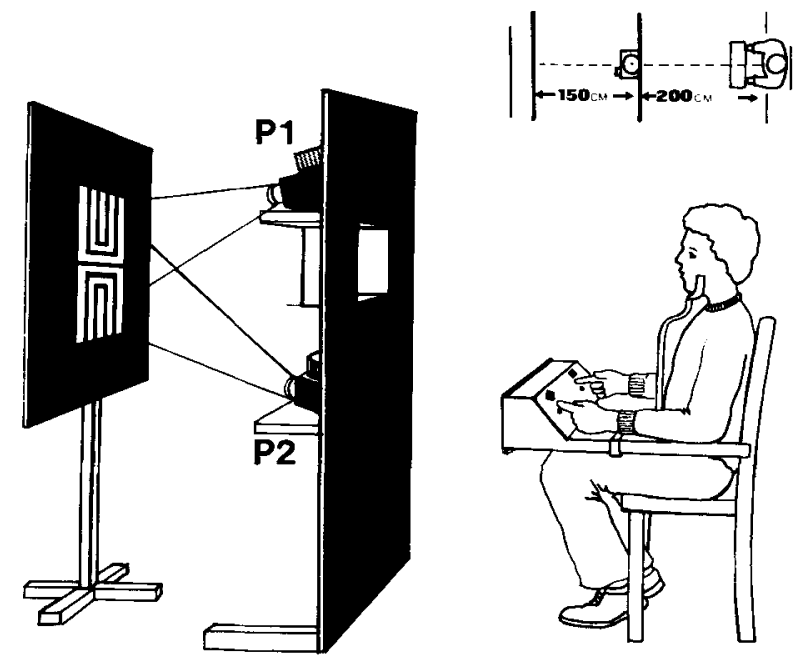

Figure 2. Schematic representation of the apparatus used in the three experiments to present stimuli and record responses. The response panel operated by the observer was connected to a Cromemco microcomputer (not shown). In Experiment 3, a footoperated switch was also in use. 
whether up or down) of each button was sampled every $100 \mathrm{msec}$ and recorded cumulatively by a Cromemco (Model Z-2D) microcomputer, thereby providing a real-time record of reported perceptual fluctuations. The microcomputer also counted the number of separate buttonpresses.

Procedure. The observers were seated in front of the stimulus display (see Figure 2) and familiarized with the apparatus. The test pattern was shown and described as an ambiguous/reversible display ("like the Necker cube"). The observers were asked to describe any reversal phenomenon experienced while they were inspecting the display. The experimenter remained silent until there was some verbal indication that observers were experiencing spontaneous reversals. By a process of questioning, the experimenter cued observers until their verbal responses contained descriptors like "diamond" or "tilted square" (for the diamond organization) and " $H$ " or "open rectangles and squares" (for the rectangles organization). The experimenter then summarized the alternation phenomenon in a standard form, explicitly using the terms "diamond" and "rectangles." Each response button was appropriately labeled, and the observers were instructed to depress the button corresponding to the dominant organization and to keep the button depressed as long as the particular perceptual organization remained dominant. The observers were instructed not to feel obliged to "see" reversals, but to relax and record any reversals as they occurred. The relationship between response button (i.e., hand) and perceived organization was counterbalanced across observers to eliminate any preferred-hand bias in responding.

Each observer was tested in two conditions, one in which the display was achromatic and one in which the display was colored. To avoid the possibility that inspection of the colored display might induce CAEs, the achromatic condition always preceded the colored condition. ${ }^{5}$ Duration of testing was 3 min for each condition with a rest interval of approximately $5 \mathrm{~min}$ between conditions.

\section{Results and Discussion}

Since observers were making a two-alternative forcedchoice response, the cumulative durations of both organizations always summed to $180 \mathrm{sec}$ (the total test time). Figure 3 shows the mean cumulative durations that the diamond and rectangles organizations were reported in the achromatic and colored conditions; the mean number of perceptual alternations in the two conditions is also shown. In the achromatic condition, duration of diamond reports (and therefore rectangles reports) was not significantly different from $90 \mathrm{sec}$, which indicates that observers were not biased in reporting one of the two per-

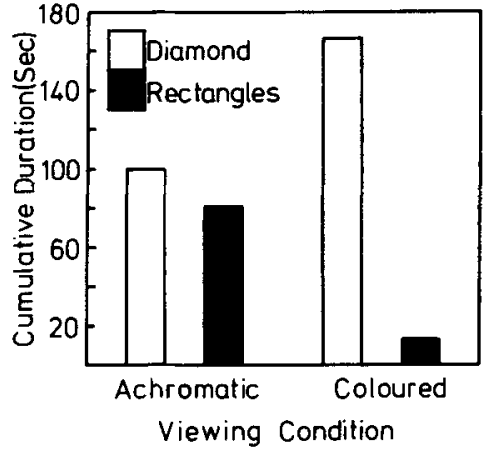

(a) ceptual organizations. A matched-pairs $t$-test indicated that the mean cumulative duration of reporting diamond increased significantly in the colored condition as relative to the achromatic condition $[t(8)=10.5, p<.001)$. Since the cumulative durations for diamonds and rectangles summed to $180 \mathrm{sec}$, the corresponding decrease in the rectangles organization was also significant. Furthermore, the mean cumulative duration for rectangles in the colored condition was not significantly different from zero $[t(8)=2.15, p>.05]$.

The results of Experiment 1 (in which real colors were added to an ambiguous test display) are in general agreement with those of Uhlarik et al. (in which CAEs were "added" to an ambiguous test display as a result of colororientation adaptation). In both cases, the nature of the ambiguous display changes when colored so that fewer perceptual alternations occur. Reports of the rectangles organization are few, and the diamond organization is reported almost exclusively. Before discussing the implications of these results for the role of CAEs and perceptual alternation phenomena in general, it is necessary to ensure that perceptual ambiguity in Figure 1b-type displays is not altered by the addition of color per se. For this reason, Experiment 2 was performed as a control for Experiment 1.

\section{EXPERIMENT 2}

The color-orientation combinations used in Experiment 1 were designed to simulate the appearance of Figure $1 \mathrm{~b}$ following successful CAE induction. In Experiment 2 , colors were added to the display in what may be described as non-CAE color-orientation combinations to determine whether the presence of color is sufficient to modify perceptual alternation of Figure $1 b$.

\section{Method}

Subjects. Eight undergraduate students who had not participated in the previous experiment volunteered to serve as observers. All were screened to meet the same requirements as those outlined for Experiment 1.

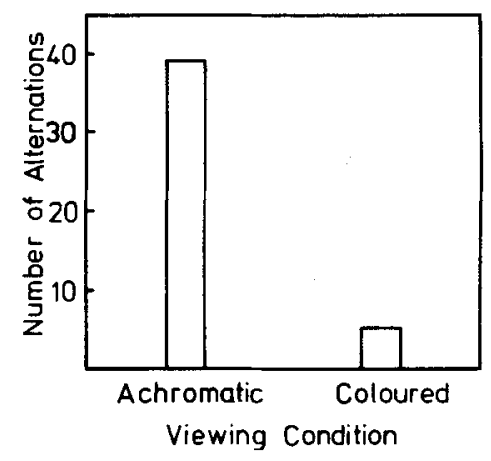

(b)

Figure 3. Mean cumulative duration (seconds) for reporting diamond and rectangles perceptual organizations in the achromatic and colored viewing conditions (a). Mean number of perceptual alternations in the two viewing conditions (b). 
Materials and Apparatus. The achromatic ambiguous test pattern and the experimental apparatus were identical to those used in the previous experiment. The test pattern was colored in the same manner except that the configuration of near-complementary colors in composite (red/green) slides was altered to meet the experimental requirements set out below. As in the previous experiment, composite slides were always defocused to eliminate any distinct $\mathrm{red} / \mathrm{green}$ boundaries. The luminances and the color saturations were also matched as closely as possible to those used in Experiment 1.

Procedure. All observers were tested in five experimental conditions. These were an achromatic control condition (C1), two conditions in which the test display was either all red or all green $(\mathrm{C} 2$ and $\mathrm{C} 3$, respectively), and two conditions in which one half of the display was red and the other half was green (C4 and C5). In C4, the display was divided along its vertical axis of symmetry (e.g., green left/red right); in $\mathrm{C5}$, the display was divided along its horizontal axis of symmetry (e.g., green top/red bottom). To avoid the effects of any aftereffects induced by the colored displays, the achromatic control condition ( $\mathrm{C} 1$ ) was always tested first. The remaining chromatic conditions were tested in counterbalanced order with the additional provision that half the observers were shown red to the left and green to the right (in $\mathrm{C} 4$ ) and red to the top and green to the bottom (in C5), and the remainder were shown the opposite color-orientation configurations. In other respects, the procedures were similar to those of Experiment 1. All conditions were tested in a single session, with rest intervals of about $5 \mathrm{~min}$ between conditions.

\section{Results and Discussion}

Separate one-way analyses of variance were performed on the cumulative durations (in seconds) of the reported diamond organization and the number of alternations in the five experimental conditions. No significant variation occurred across the five conditions for either duration $[F(4,28)<1.0]$ or alternation $[F(4,28)<1.0]$. The mean cumulative durations for diamond reports and the mean number of alternations in the five experimental conditions were highly consistent with the values obtained during the achromatic condition of Experiment 1. To enable comparisons, the data obtained in Experiment 2 are shown in Table 1 .

Taken together, the results of Experiments 1 and 2 indicate that the ambiguous nature of Figure $1 \mathrm{~b}$ is modified by the addition of colors, but only when the added colors are similar to the appearance of CAEs. When the horizontal and vertical components of Figure $1 \mathrm{~b}$ are colored with near-complementary colors, Figure $1 \mathrm{~b}$ becomes an unambiguous display with the diamond configuration being reported almost exclusively. Observers' responses in $\mathrm{C} 2$ and $\mathrm{C} 3$ of Experiment 2 (i.e., in conditions in which the display was viewed in homogeneous

Table 1

Mean Duration of Diamond Reports and Mean Number of Alternations (Together With Standard Errors in Parentheses) for the Five Experimental Conditions of Experiment 2

\begin{tabular}{llllll}
\hline & \multicolumn{5}{c}{ Experimental Conditions } \\
\cline { 2 - 4 } & $\mathrm{C} 1$ & $\mathrm{C} 2$ & $\mathrm{C} 3$ & $\mathrm{C} 4$ & $\mathrm{C} 5$ \\
\cline { 2 - 6 } Cumulative & 94.7 & 86.7 & 89.5 & 88.7 & 90.1 \\
Durations (Sec) & $(3.8)$ & $(5.8)$ & $(5.2)$ & $(6.8)$ & $(7.6)$ \\
Number of & 41.0 & 43.0 & 42.4 & 39.8 & 40.5 \\
Alternations & $(4.2)$ & $(2.3)$ & $(1.6)$ & $(2.0)$ & $(2.5)$ \\
\hline
\end{tabular}

red and green light, respectively) were almost identical to responses given when the display was viewed in homogeneous white light. These conditions can be compared to the zero-disparity dichoptic viewing conditions used by Lawson et al. (1977), in which no change in perceptual alternation was found. That is, neither the viewing of Figure $1 \mathrm{~b}$ under dichoptic conditions nor the viewing of Figure $1 \mathrm{~b}$ under conditions of narrow band illumination are sufficient, per se, to disambiguate the display: For this to occur, particular values of binocular disparity are necessary in the former case, and particular color-orientation combinations are necessary in the latter. ${ }^{6}$

The results of Experiments 1 and 2 indicate that the procedures used to make ambiguous displays unambiguous are not as straightforward as they may appear, and clearly warrant further investigation. More importantly, however, the results call into question the most commonly made interpretation of the relationship between perceptual aftereffects and perceptual alternation, namely that the former phenomena are under the control of the same mechanisms that determine the latter (see Broerse \& Crassini, 1981). If the addition of CAE-like colors can alter an observer's perception of an ambiguous display, then it is likely that the production of CAEs can produce a similar result. This expectation was tested in Experiment 3.

\section{EXPERIMENT 3}

The argument that CAEs (and motion aftereffects; Meyer \& Sherman, 1981) depend on the perceived organization of an ambiguous test figure rests on the assumption that the procedures that produce the aftereffects and their outcomes do not alter perceptual organization. Despite the crucial nature of this assumption, most of the studies purporting to demonstrate the dependence of aftereffects on perceptual organization either ignored completely the issue of perceptual organization following aftereffect-adaptation or used procedures not suitable for its evaluation. In three of the studies (i.e., Jenkins \& Ross, 1977; Meyer \& Phillips, 1980; Meyer \& Sherman, 1981) neither the frequency of alternation between perceived organization nor fluctuations (e.g., presence/absence) in the appearance of aftereffects was measured. Data reported in these articles were based on observers' introspective verbal reports about the color appearance and perceptual organization of the test display. Meyer and Phillips (1980) did report the frequency of perceptual alternation in a pilot study, but did not provide similar data following colororientation adaptation.

Using an entirely different procedure, Milewski et al. (1980) induced CAEs in the normal manner and asked observers to rate CAE strength (on an 11-point scale) while inspecting (1) orthogonal gratings (i.e., a standard CAE test pattern), (2) two checkerboard patterns, and (3) a set of concentric octagons (which could be perceived either as a set of concentric octagons or as a set of eight triangular wedges). In addition, observers were asked to rate (on a 4-point scale) the ease with which they could 
switch consciously from the organization perceived spontaneously in the test display to the alternative organization. Data reported with the octagon display are of special relevance to the present argument. Prior to adaptation, the 34 observers were almost equally divided in terms of their spontaneously reported perceptual organization (19 reported octagons; 15 reported triangles). However, following adaptation, only 9 observers reported octagons and 25 reported triangles. Moreover, CAEs were rated as stronger during the spontaneous organization than during the alternative. In addition to producing CAEs and a shift in the distribution of spontaneously perceived organization, adaptation produced a change in the reported difficulty of switching from one perceptual organization to the other; the 25 observers who reported spontaneously the triangles organizations and CAEs after adaptation also reported the greatest difficulty in switching to the alternative organization of octagons.

The data reported by Milewski et al. (1980) are complex and somewhat difficult to interpret. For example, observers who were unable to switch their perceptual organization, or who experienced great difficulty in doing so, were still required to give a subjective CAE rating for the alternative perceptual organization. Despite these problems, the data provide support for the idea that adaptation influences both the color appearance and perceptual organization of an ambiguous test display. However, the procedures used by Milewski et al. (1980) do not address this issue directly. They also introduce a new set of problems concerning the ability of observers to reorganize ambiguous figures at will, and to rate both the effort required in this task and the appearance of the new organization. The techniques most suited to answering questions about changes in perceived organization following adaptation are the continuous real-time monitoring procedures used by Uhlarik et al. (1977). Unfortunately, these authors used an inappropriate index to summarize their data on perceived organization, and did not use the same procedures to monitor CAEs (see Broerse \& Crassini, 1981). As in the other studies mentioned above, inferences about fluctuations in CAE strength and perceptual alternations were based on observers' introspections at the completion of testing.

In Experiment 3, the continuous recording facilities used in Experiments 1 and 2 were modified so that fluctuations in perceptual organization and CAE presence could be measured. Furthermore, a critical test of the assumption that color-orientation adaptation alters both the color appearance and perceptual organization of an ambiguous test display was carried out. It is characteristic of CAEs that they do not transfer interocularly in a simple fashion (see Stromeyer, 1978, for a review of this issue). Therefore, if adaptation influences perceived organization, it should be possible to elicit two separate perceptual alternation rates in the same observer by monocular color-orientation adaptation. When looking at the ambiguous test display with the nonadaptation eye, fluctuations in perceptual organization should be similar to those reported before adaptation when viewing with either eye. However, when using the adaptation eye, the test display should appear colored and the diamond organization should predominate just as in the simulatedCAE condition of Experiment 1.

\section{Method}

Subjects. A further 8 undergraduate students with normal or corrected-to-normal visual acuity (and who had not participated in Experiments 1 and 2) volunteered to serve as observers. All were naive as to the purpose of the experiment and were screened for optical astigmatism and color vision defects, as in the previously reported experiments.

Materials and Apparatus. A Kodak Carousel projector (P1, see Figure 2) was used to project adaptation stimuli onto a white rectangular screen with a matte black surround. The stimuli consisted of horizontal and vertical square-wave gratings $(4.2$ cycles $/$ deg of visual angle) that formed a square with sides subtending $2.8^{\circ}$ of visual angle. Kodak Wratten filters were used to produce red (No. 26) vertical and green (No. 55) horizontal gratings. The achromatic ambiguous test pattern was the same as that used in the experiments reported above, and its spatial frequency was identical to the spatial frequency of the adaptation stimuli.

The experimental apparatus shown in Figure 2 and described in Experiment 1 was used to present adaptation/test stimuli and to record responses. A footswitch was also provided so that observers could report the presence of aftereffect colors. A standard laboratory interval generator was used to control the presentation of stimuli.

Procedure. Each observer participated in a pretest, an adaptation, and a posttest session. Four observers adapted with the left eye and four observers adapted with the right eye. The observers wore a standard trial-lens spectacle frame so that a black occluding disk could be inserted during the appropriate adaptation and test conditions. When required, optometrically prescribed correction lenses could also be inserted. The order of testing for adaptation and nonadaptation eyes was counterbalanced over adaptation and test conditions (e.g., when observers were required to adapt with their left eyes, half were tested with the order right eye/left eye). In addition, 4 observers received the same orders for pretest and posttest; for the remaining 4 observers the orders were reversed. During both test sessions, the observers were required to inspect the same ambiguous test figure used in Experiments 1 and 2 and report, by pushing response buttons in the manner described above (i.e., for the diamond or rectangles organizations), which organization was dominant. For posttest conditions, the visibility of CAEs was monitored by a footswitch which observers were required to keep depressed whenever CAEs were visible. During induction, observers inspected, in alternation, red vertical and green horizontal gratings for $20 \mathrm{~min}$. Each grating was presented for $8.5 \mathrm{sec}$ and was followed by a dark interval of $1.5 \mathrm{sec}$. Responses during test sessions were recorded as in Experiments 1 and 2, and could be monitored by the experimenter on a small video display situated in an adjacent room. If the footswitch for recording color responses was depressed at any time during posttest, the observers were interrogated about the nature of the color appearance at the conclusion of the posttest session; they were asked to indicate which colors were seen and whether colors were associated with particular regions of the test pattern.

\section{Results and Discussion}

Initially, the data on perceived organization and perceptual alternation were analyzed independently of color reports. Two separate two-way repeated measures analyses of variance were performed on, respectively, the cumulative duration of the diamond organization and the 
total number of alternations. The two factors in these analyses were (1) viewing condition (pretest vs. posttest), and (2) inspection eye (adaptation eye vs. nonadaptation eye).

The cumulative duration for the diamond organization varied significantly as a function of viewing condition $[F(1,7)=90.24, p<.001]$ and inspection eye $[F(1,7)$ $=60.79, p<.001]$. The interaction between these factors was significant $[F(1,7)=34.67, p<.001]$, and subsequent comparisons between individual means (see Figure 4a) showed that the duration of reporting diamond with the adaptation eye was significantly greater during the posttest than during the pretest $[t(7)=8.37$, $p<.001]$. There were no differences between pretest and posttest scores for the nonadaptation eye, and neither differed significantly from the pretest duration in the adaptation eye. The total duration for the diamond organization during posttest was significantly greater for the adaptation eye than for the nonadaptation eye $[t(7)=14.94$, $p<.001]$. Since the cumulative durations for the diamonds and rectangles organizations summed to $180 \mathrm{sec}$, the corresponding decreases in the rectangles organization in all the above comparisons were significant also. In addition, the mean cumulative duration for the rectangles condition in the adaptation eye (posttest) was not significantly different from zero [mean $=10.1 \mathrm{sec} ; t(7)=$ $1.67, p>.05]$. Mean durations for periods when CAEs were not reported, both when the figure was seen in the diamond organization (mean $=7.6 \mathrm{sec}$ ) and when it was seen in the rectangles organization (mean $=7.5 \mathrm{sec}$ ), were also not significantly different from zero [both $t \mathrm{~s}(7)$ $=1.1, p>.05]$.

The change in reported duration of the diamond organization following monocular chromatic adaptation was associated with a change in the number of perceptual alternations. These varied significantly as a function of viewing condition $[F(1,7)=23.11, p<.003]$ and inspection eye $[F(1,7)=55.23, p<.001]$. The interaction was significant $[F(1,7)=376.12, p<.001]$. Inspection of Figure $4 \mathrm{~b}$ shows that pretest alternation rates when either eye was used were not significantly different from each other, nor was either value different from the posttest alternation rate when the nonadaptation eye was used. The posttest alternation rate when the adaptation eye was used was significantly less than the pretest rate with the same eye $[t(7)=8.43, p<.003]$. In other words, monocular color-orientation adaptation resulted in an increase in the duration of reporting the diamond organization and a decrease in alternation rate, but only when testing with the adaptation eye.

Color responses and perceptual alternation responses were treated concurrently by calculating the proportions of time CAEs were reported during periods of dominance of the diamond organization and rectangles organization separately. In the adaptation eye (posttest), CAEs were reported for $95.4 \%$ of the time that the diamond organization (D) was dominant, and for only $25.7 \%$ of the time that the rectangles organization $(R)$ was dominant-that is, the conditional probabilities for the aftereffects (A) were $p(\mathrm{~A} / \mathrm{D})=.95$ and $p(\mathrm{~A} / \mathrm{R})=.26$. This pattern of $\mathrm{CAE}$ reports and perceptual organization responses seems to be consistent with the view that CAEs are contingent not only on contour orientation but also on perceptual organization. However, this interpretation is misleading. It does not take into account the large increase in the time the test display is seen in the diamond organization and the concomitant reduction in the number of alternations following adaptation (see Figure 4). These increases in perception of the diamond organization were such that perception of the rectangles organization was almost completely absent (i.e., mean duration of $\mathbf{R}$ was not significantly different from zero). Accordingly, $25.7 \%$ of time that aftereffects were present during the rectangles organization was also not significantly different from zero.

An appropriate indication of the postadaptation changes described above is found by considering the conditional probabilities for reporting diamond in the presence and in the absence of aftereffects [i.e., $p(\mathrm{D} / \mathrm{A})$ and $p(\mathrm{D} / \overline{\mathrm{A}})$, respectively]. During posttest of the adaptation eye, the

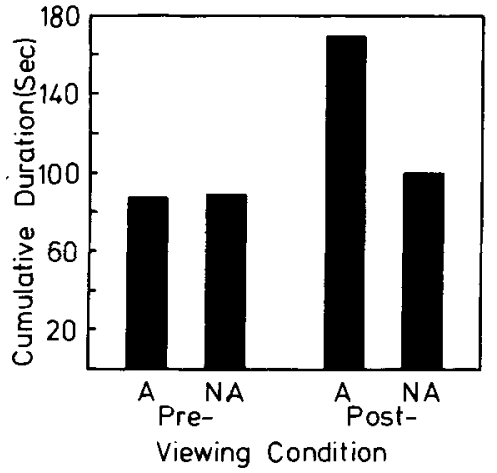

(a)

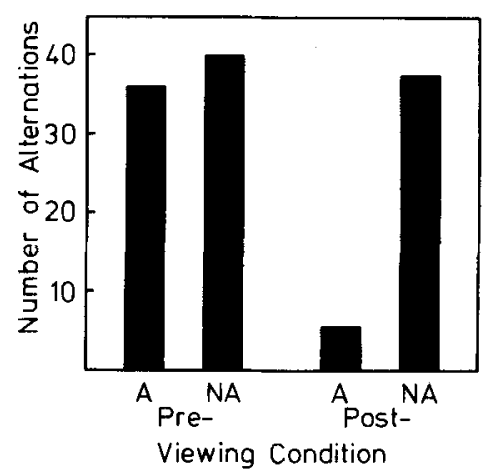

(b)

Figure 4. Mean cumulative duration (seconds) for reporting diamond perceptual organization before (pre-) and after (post-) monocular color-orientation adaptation. Separate means are shown for viewing with the adaptation (A) eye and nonadaptation (NA) eye (a). Mean number of perceptual alternations before and after adaptation are also shown for the adaptation and nonadaptation eyes (b). 
diamond organization was reported for $98 \%$ of the time that aftereffects were visible $[p(D / A)=.98]$ and for $50 \%$ of the time that aftereffects were not visible $[p(\mathrm{D} / \overline{\mathrm{A}})=$ $.50]$. Although the durations for $\bar{A}$ were not significantly different from zero, it is worthwhile noting that the value of $p(\mathrm{D} / \overline{\mathrm{A}})$ was comparable to $p(\mathrm{D})$ in all conditions in which CAEs were not present or expected [i.e., pretest, adaptation eye, $p(\mathrm{D})=.50$; pretest, nonadaptation eye, $p(\mathrm{D})=.50$; posttest, nonadaptation eye, $p(\mathrm{D})=.55]$. In light of the postadaptation changes described (which occurred only during testing with the adaptation eye), the interpretation that CAEs are more likely to be reported during dominance of the diamond organization (e.g., Uhlarik et al., 1977) is in a sense trivial, since only the diamond organization tends to be reported after adaptation.

\section{GENERAL DISCUSSION}

We had three aims in completing the experiments described above. The first was to clarify the relationship between CAEs and changes in perceived organization (perceptual alternation). The second was to examine the implications of this relationship for neurally based accounts of CAEs, ambiguous displays like Figure $1 \mathrm{~b}$, and related examples of perceptual fluctuations (e.g., monocular rivalry). The third was to consider these specific implications within the broader theoretical contexts of direct and indirect approaches to perception.

As indicated in the introduction, the most commonly held interpretation of the relationship between CAEs and perceptual alternation is that both phenomena are under the control of some central cognitive process. It was this process that mediated the imposition of a particular organization on an ambiguous display, and in so doing determined the appearance (or disappearance) of CAEs (see Broerse \& Crassini, 1981). Our argument is that both CAEs and perceptual alternation are stimulus bound. The data of Experiments 1 and 2 (see Figures 2 and 3 and Table 1) clearly support this argument in that the addition of separate colors to the horizontal and vertical components of Figure $1 \mathrm{~b}$ make the display unambiguous. Furthermore, the results of Experiment 3 (see Figure 4) indicate that the process of disambiguating ambiguous displays need not involve changes to the displays themselves. It is sufficient to alter the functioning of the mechanisms (within the observer) assumed to be involved in processing the displays. For example, McCollough-type adaptation alters color-orientation processing mechanisms with the result that an achromatic ambiguous display (with appropriate spatial characterisitics, e.g., Figure 1b) appears to be colored for a period of time following adaptation. During this period, observers respond as if the display were colored, and the display is no longer perceived as being ambiguous. Taken together, the data of Experiments 1,2 , and 3 suggest that the common interpretation of the relationship between CAEs and perceptual alternation should be reversed. Rather than perceptual organization's determining CAE appearance or disappearance, the occurrence of CAEs determines perceptual organization.

This causal interpretation is strengthened by the interocular comparisons possible in Experiment 3. The monocular color-orientation adaptation procedures resulted in the manifestation of CAEs only when testing took place with the adaptation eye. Thus, the same observer reported quite distinct color experiences and perceptual alternation experiences depending on the eye used during adaptation and test. This indicates that the adaptation procedure alone is not sufficient to alter the perceptual alternation experiences of the observer. It is only when these adaptation procedures produce CAEs that changes in perceptual alternation occur. In summary, our first aim was achieved. We have shown that perceptual alternation of Figure $1 \mathrm{~b}$ is contingent on the color appearance of the display, irrespective of whether the color appearance is the result of changes in illumination wavelength or is due to color-orientation adaptation of the observer.

The finding that the rectangles organization is almost never reported when complementary colors (real or aftereffect) are paired with the orthogonal contours of Figure $1 \mathrm{~b}$ is consistent with findings that the composite organization during monocular rivalry also is decreased markedly when such colors are paired with orthogonal contours (see above). Fluctuations in the visibility of contours during monocular rivalry are attributed to inhibitory interactions between populations of orientationselective mechanisms in the visual cortex (e.g., Morrone et al., 1982). Such mechanisms exhibit color selectivity and are assumed to account for changes in the nature and rate of monocular rivalry when colored contours are used (Rauschecker et al., 1973; Stromeyer, 1978). But these same color-orientation mechanisms (Gouras \& Kruger, 1979; Gouras \& Zrenner, 1981) are most commonly assumed to account for CAEs (see Harris, 1980; Over, 1977; Stromeyer, 1978). In relation to our second aim, therefore, we suggest that our results may be interpreted in a manner that achieves a considerable degree of parsimony: The mechanisms underlying perceptual fluctuations during monocular rivalry, and during inspection of Figure $1 \mathrm{~b}$, are the same mechanisms that underlie CAEs when Figure $1 \mathrm{~b}$ and similar ambiguous figures are used as CAE-test stimuli.

Our third aim concerned the indirect theoretical context adopted implicitly in previous interpretations of the relationship between CAEs and perceptual alternation of ambiguous displays like Figure $1 \mathrm{~b}$. That is, the interpretation that the cognitive processes that impose order on (and determine perceptual organization of) ambiguous sensory input provided by ambiguous displays also cause the test display to change in color appearance. When complementary colors were superimposed on the test figure (irrespective of whether they were real or aftereffect colors), there were no longer any significant changes in perceived organization. Strictly speaking, this leaves the question of whether changes in perceived organization influence CAEs unanswerable (i.e., there were no changes). 
However, if such changes no longer occur, there would appear to be little sense left in the claim that such changes (and the cognitive factors responsible for them) cause fluctuations in the appearance of CAEs. The results reported in this paper suggest that such an interpretation (and its implicit neorationalist framework) is not necessary to explain how CAEs and perceptual alternation may be related. Indeed, the present results are consistent with what we have termed neuroempiricist approaches to perception. That is, the data reported here are consistent with the proposition that the neural mechanisms mediating the occurrence of CAEs are also involved in the fluctuations in perceptual organization found with ambiguous displays.

The term neoempiricist is used by Uttal (1981) to encompass Gibson's theory of perception, since Gibson also attributes perception solely to stimulation from the natural environment (or more correctly, to an interaction between the observer and environmental stimulation). Gibson's (1979) proposals about the relationship between information available in stimulation from natural, threedimensional environments and the information available in pictorial arrays are useful in describing the conditions that define perceptual ambiguity in displays like Figures $1 \mathrm{a}$ and $\mathbf{1 b}$. For example, the addition of texture gradient information to Figure 1a or particular color information to Figure $1 \mathrm{~b}$ renders both displays unambiguous. However, the utility of Gibson's approach in explaining perceptual ambiguity diminishes beyond this point. For Gibson, interpretations (or perceptions) of ambiguous displays are ambiguous because the information to be interpreted is ambiguous. Despite the argument that such situations arise only in "unnatural" contexts (e.g., pictures; see Gibson, 1966, 1979, and Michaels \& Carello, 1981), and that they therefore contribute little to the understanding of real-world perception, there is very little to distinguish Gibson's approach to perceptual ambiguity from the more traditional (indirect) approaches to perception. Irrespective of whether information in pictures is taken to consist of two-dimensional simulations of Gibsonian invariant relations, a patchwork of Wundtian sensations, or Gestalt-like figures and forms, perception, in each case, is based on some form of interpretation.

What does distinguish Gibson's approach to perception from those of others is that the analysis just outlined (i.e., perception as interpretation) is taken by Gibson to refer only to the perception of pictures and not to the perception of the real world. However, whether the "perceptionas-interpretation"' approach is adopted only when discussing perception of pictures or is used when discussing perception in general, the question remains: Can these higher order, interpretative processes influence the functioning of the lower order (stimulus-bound) processes on whose output they are assumed to operate? The data of the three experiments reported here suggest not. The lower order mechanisms taken to determine the occurrence of CAEs were not influenced by higher order mechanisms assumed to determine perceptual organization. Rather, the present data indicate that the reverse is more likely to be the case.
In none of the theoretical approaches considered above is the notion that perception is stimulus driven-that what is seen depends on what it is that is seen-in dispute. ${ }^{7}$ There is even provision in some indirect (i.e., neorationalist) approaches for stimulus-driven mechanisms to be stimulus-bound to some extent. Such mechanisms are assumed to be autonomous (i.e., cognitively impenetrable; Fodor \& Pylyshyn, 1981) yet capable of processing quite complex perceptual aspects of a stimulus array. Furthermore, depending upon how perception is defined (i.e., what is considered to be visual perception and what is considered to be visual cognition; see Dretske, 1978), there is little dispute concerning the notion that what something is seen as depends on some higher order cognitive processing (i.e., interpretation, prior experience, knowledge), even if this is taken only to apply to the perception of pictures. A dispute does arise, however, with respect to the question of whether (and in what circumstances) the processes determining what something is seen as influence the processes determining what is seen (i.e., whether the latter are cognitively penetrable; Fodor \& Pylyshyn, 1981). In these terms, the evidence provided by our investigations of ambiguous displays like Figure $1 \mathrm{~b}$ suggests that the mechanisms that determine which perceptual organization is seen are the same as the mechanisms involved in processing the color/spatial components of these displays. Furthermore, such mechanisms are cognitively impenetrable with respect to the processes that determine what these organizations are seen as.

A final point regarding Gibsonian approaches warrants consideration. According to exponents of Gibson's approach, distinctions between seeing and seeing as, between cognitively impenetrable and cognitively penetrable processes, are examples of a "dualism" between perceiver and environment inherent in traditional indirect accounts of perception (see Michaels \& Carello, 1981). Such dualisms form the basis of what is commonly referred to as the classical problem in perceptual theory (e.g., Wertheimer, 1974)-namely the difficulty in defining the properties of what is seen in terms independent of what things are seen as. Spots, edges, Gestalt-like figures, are the objects of perception (i.e., the functional outcomes for a perceiver), not properties of things themselves. To overcome this problem, Gibson (1979) advocates that such dualisms be avoided and perception considered in terms of an interaction between perceiver and environment. What is directly "picked up" from the environment is not a collection of edges, angles, and so forth, that are indirectly perceived as, say, a stick, but the property that a stick affords a perceiver (i.e., its "'graspability"). Although properties like "graspability" are clearly interactional in nature, it seems to us that they focus as much on the functional outcomes of perception as do the neorationalist approaches that Gibsonians attempt to refute. Although we agree that any comprehensive theory of perception should be based on considerations involving interactions between perceiver and environment, we believe that the role of the perceiver's nervous sys- 
tem cannot be ignored in such considerations. We take the data reported in Experiment 3 to support this point of view.

In conclusion, the results of the experiments reported show that appropriate addition of color (whether physically present or induced as CAEs) to an ambiguous display is sufficient to disambiguate the display. These results are not consistent with the proposal that cognitive factors influence the stimulus-bound mechanisms responsible for CAEs. Rather, they can be understood in terms of the activity of neural mechanisms common to CAEs and perceptual alternation.

\section{REFERENCES}

Attneave, F. (1976). Multistability in perception. In R. Held \& W. Richards (Eds.), Recent progress in perception. San Francisco: Freeman.

Benevento, L. A., Creutzfeldt, O. D., \& Kuhnt, V. (1972). Significance of intracortical inhibition in the visual cortex. Nature, 238, 124-126.

Blakemore, C., \& Tobin, E. A. (1972). Lateral inhibition between orientation detectors in the cat visual cortex. Experimental Brain Research, 15, 439-440.

Broerse, J., \& Crassini, B. (1981). Comments on the use of perceptually ambiguous figures as McCollough-effect stimuli. Perception \& Psychophysics, 30, 339-402.

Burr, D. C., Morrone, C., \& MAFFeI, L. (1981). Intracortical inhibition prevents simple cells from responding to textured patterns. $E x$ perimental Brain Research, 43, 455-458.

Campbell, F. W., Gilinsky, A. S., Howell, E. R., Riggs, L. A., \& ATKINSON, J. (1973). The dependence of monocular rivalry on orientation. Perception, 2, 123-125.

DreTsKe, F. (1978). The role of the percept in visual cognition. In C. W. Savage (Ed.), Perception and cognition: Issues in the foundations of psychology. Minneapolis: University of Minneapolis Press.

EMmerson, P. L. (1979). Necker cube: Duration of preexposure of an ambiguous form. Bulletin of the Psychonomic Society, 14, 397-400.

EsCHER, M. C. (1961). The graphic art of M. C. Escher. London: Oldbourne.

Fodor, J. A., \& Pylyshyn, Z. W. (1981). How direct is visual perception? Some reflections on Gibson's 'ecological approach.' Cognition, 9, 139-196.

GiBson, J. J. (1966). The senses considered as perceptual systems. Boston: Houghton Mifflin.

GiBson, J. J. (1979). The ecological approach to visual perception. Boston: Houghton Mifflin.

Gouras, P., \& KRUger, J. (1979). Responses of cells of foveal visual cortex of the monkey to pure color contrast. Journal of Neurophysiology, 42, 850-860.

Gouras, P., \& ZRENNER, E. (1981). Color vision: A review from a neurophysiological perspective. In D. Ottoson, H. Autrum, E. R. Perl, \& R. F. Schmidt (Eds.), Progress in sensory physiology I. Berlin: Springer-Verlag.

GrEGORY, R. L. (1974). Choosing a paradigm for perception. In E. C. Carterette \& M. P. Friedman (Eds.), Handbook of perception (Vol. 1). New York: Academic Press.

GREGORY, R. L., \& GOMBRICH, E. H. (Eds.) (1973). Illusion in nature and art. London: Duckworth.

Harris, J. P. (1979). The Schroeder staircase: A new perspective. Perception \& Psychophysics, 26, 312-318.

Harris, C. S. (1980). Insight or out-of-sight? Two examples of perceptual plasticity in the human adult. In C. S. Harris (Eds.), Visual coding and adaptability. Hillsdale, NJ: Erlbaum.

Hering, E. (1964). Outlines of a theory of the light sense (L. M. Hurvich \& D. Jameson, Trans.). Cambridge, MA: Harvard University Press. (Original work published 1874)

Jenkins, B. , \& Ross, J. (1977). McCollough effect depends upon perceived organizations. Perception, 6, 399-400.
Lawson, R. B., Packard, S., LaWrence, D., \& Whitmore, C. L. G. (1977). Stereopsis from reversible and irreversible patterns. Perception \& Psychophysics, 21, 65-68.

McCollough, C. (1965). Color adaptation of edge detectors in the human visual system. Science, 149, 1115-1116.

Meyer, G. E., \& Phillips, D. (1980). Faces, vases, subjective contours and the McCollough effect. Perception, 9, 603-606.

Meyer, G. E., \& Sherman, R. H. (1981). Reversible figures and the motion aftereffect. Vision Research, 21, 361-363.

Michaels, C. F., \& Carello, C. (1981). Direct perception. Englewood Cliffs, NJ: Prentice-Hall.

Milewski, A. E., Iaccino, J., \& Smith, D. (1980). Checkerboardspecific color aftereffects: A failure to find effects of perceptual organization. Perception \& Psychophysics, 28, 329-336.

Morrone, M. C., Burr, D. C., \& MaffeI, L. (1982). Functional implications of cross-orientation inhibition of cortical visual cells. I Neurophysiological evidence. Proceedings of the Royal Society. London. B.216, 335-354.

OATLEY, K. (1978). Perceptions and representations. London: Methuen. OVER, R. (1977). Contingent aftereffects in the perception of colour and contour. In R. H. Day \& G. V. Stanley (Eds.), Studies in perception. Perth: University of Western Australia Press.

Rauschecker, J. P. J., Campbell, F. W., \& Atkinson, J. (1973). Color opponent neurones in the human visual system. Nature, 245, $42-43$.

STROMEYer, C. F. (1978). Form-color aftereffects in human vision. In R. Held, H. W. Leibowitz, \& H. L. Teuber (Eds.), Handbook of sensory physiology (Vol. 8). Heidelberg: Springer-Verlag.

Turvey, M. T., Shaw, R. E., Reed, E. S., \& Mace, W. M. (1981). Ecological laws of perceiving: In reply to Fodor and Pylyshyn. Cognition, 9, 237-304.

Uhlarik, J., Pringle, R., \& Brigell, M. (1977). Color aftereffects contingent on perceptual organization. Perception \& Psychophysics, 22, 506-510.

UTTAL, W. R. (1981). A taxonomy of visual processes. Hillsdale, NJ: Erlbaum.

VIRSU, V. (1975). Determination of perspective reversals. Nature, 257, 786-787.

WADE, N. J. (1975). Monocular rivalry between contours. Perception, 4, 85-95.

WADE, N. J. (1977). Distortions and disappearances of geometrical patterns. Perception, 6, 407-433.

WADE, N. J., \& DAY, R. H. (1978). On the colors seen in achromatic patterns. Perception \& Psychophysics, 23, 261-264.

WERTHEIMER, M. (1974). The problem of perceptual structure. In. E. C. Carterette \& M. P. Friedman (Eds.), Handbook of perception (Vol. 1). New York: Academic Press.

\section{NOTES}

1. The configuration most commonly reported for the diamond organization of Figure $1 \mathrm{~b}$ is that in which the diamond-shaped horizontal contours are reported as "figure" against a "ground" of vertical contours (see Uhlarik, Pringle, \& Brigell, 1977). However, it may be possible also to see a rectangular figure (vertical contours) that reveals a ground of horizontal contours through a diamond-shaped "hole." To the best of our knowledge, no one has ever investigated whether this reversed configuration is ever reported.

2. This is not to imply that perceptual "errors" should not occur, in Gibsonian terms, in the natural environment. Indeed, some types of perceptual "error" (e.g., a stick appearing to bend when immersed in water) are predicted in Gibson's theory, given the properties of the environment and the perceiver. (See Turvey, Shaw, Reed, \& Mace, 1981, for a discussion of the relevance of misperceptions to Gibson's theory of perception.)

3. All observers were also informally screened for astigmatism in both horizontal and vertical axes. The need for this became obvious when, during piloting, I observer (who was not a participant in the present experiment) was unable to see the rectangles organization at all when the achromatic test figure was presented. When subsequently shown a set of concentric squares (the figure resembling that used by Jenkins 
\& Ross, 1977) and asked to comment on the clarity of the lines, this subject said that the vertical component always appeared blurred. Since the Jenkins and Ross figure itself resembles a pattern used by Verhoeff (1799, cited in Wade \& Day, 1978) as a test of astigmatism, all subjects in each of the three experiments were subjected to this screening procedure. We are currently preparing a paper on the effect of artificially induced astigmatism (in the horizontal and vertical axes) on the appearance of the figures used in the present experiment.

4. Both authors judged the effect to be a reasonably good facsimile of CAEs. However, since the saturation of both red and green was not controlled independently, a setting which provided a green that was comparable to the green commonly experienced with CAEs also tended to produce a red that was marginally more pronounced than that experienced with CAEs. By way of anecdote, the simulation was sufficiently convincing to momentarily deceive two independent and disinterested members of staff (who had previously participated as observers in a CAE demonstration) into believing that they were still experiencing CAEs generated 3 to 4 months earlier.

5. Although this procedure does not involve counterbalancing to remove any possible order effects, it represents a conservative design weakness. The responses made during viewing of the achromatic display (i.e., a steady rate of perceptual alternation and almost equal durations of perceptual dominance of the two organizations) in Session 1 are not in the direction expected during Session 2 (i.e., when viewing the colored ver- sion of the display). Any expectations or response biases developed during Session 1 are therefore working against the expected effects.

6. The colored displays of $\mathrm{C} 4$ and $\mathrm{C} 5$ may be characterized as containing a further source of ambiguity when compared with the achromatic version of Figure $1 \mathrm{~b}$. Together with the diamond and rectangles organizations, the colored display might also be organized as containing two adjacent colored surfaces with either a vertical (C4) or a horizontal (C5) border. These additional colored surfaces, however, do not coincide with either the diamond or rectangles organization (which observers were asked to report). For this reason, presumably, observers' reports of perceptual organization in these conditions were no less ambiguous than in the three homogeneous illumination viewing conditions $(\mathrm{Cl}, \mathrm{C} 2$, and $\mathrm{C} 3$ ).

7. A simple illustration of the difference between "seeing" and "seeing as" is provided in Footnote 3. Our (discarded) subject with severe astigmatism in the vertical axis never saw what subjects with normal vision could see as the rectangles organization. Nor would a hypothetical observer, with no previous experience or knowledge as to what sort of information constitutes a rectangle or a diamond, be able to report that what he or she was at present seeing was as (or like) diamonds or rectangles normally appear.

(Manuscript received June 3, 1985; revision accepted for publication February 4, 1986.) 